\title{
Role of core promoter sequences in the mechanism of swarmer cell-specific silencing of gyrB transcription in Caulobacter crescentus Jennifer C England and James W Gober*
}

Address: Department of Chemistry and Biochemistry and Molecular Biology Institute, University of California, Los Angeles Los Angeles, CA, 90095-1569, USA

Email: Jennifer C England - england@chem.ucla.edu; James W Gober* - gober@chem.ucla.edu

* Corresponding author

Published: 17 May 2005

BMC Microbiology 2005, 5:25 doi:10.1/86/147|-2180-5-25
Received: 01 March 2005

Accepted: 17 May 2005

This article is available from: http://www.biomedcentral.com/I47/-2/80/5/25

(c) 2005 England and Gober; licensee BioMed Central Ltd.

This is an Open Access article distributed under the terms of the Creative Commons Attribution License (http://creativecommons.org/licenses/by/2.0), which permits unrestricted use, distribution, and reproduction in any medium, provided the original work is properly cited.

\begin{abstract}
Background: Each Caulobacter crescentus cell division yields two distinct cell types: a flagellated swarmer cell and a non-motile stalked cell. The swarmer cell is further distinguished from the stalked cell by an inability to reinitiate DNA replication, by the physical properties of its nucleoid, and its discrete program of gene expression. Specifically, with regard to the latter feature, many of the genes involved in DNA replication are not transcribed in swarmer cells.

Results: We show that for one of these genes involved in DNA replication, gyrB, its pattern of temporal expression depends upon an 80 base pair promoter region with strong resemblance to the Caulobacter crescentus $\sigma^{73}$ consensus promoter sequence; regulation does not appear to be affected by the general strength of the promoter activity, as mutations that increased its conformity with the consensus did not affect its cell-cycle expression pattern. Transcription from the gyrB promoter in vitro required only the presence of the $\sigma^{73}$ RNA polymerase (from E. coli) and the requisite nucleoside triphosphates, although a distinct binding activity, present in crude whole-cell extracts, formed a complex gyrB promoter DNA. We also assayed the effect on gyrB expression in strains containing mutations in either smc or $\mathrm{dps}$, two genes encoding proteins that condense DNA. However we found there was no change in the temporal pattern of gyrB transcription in strains containing deletions in either of these genes.

Conclusion: These experiments demonstrate that gyrB transcription does not require any auxiliary factors, suggesting that temporal regulation is not dependent upon an activator protein. Swarmer-specific silencing may not be attributable to the observed physical difference in the swarmer cell nucleoid, since mutations in either smc or dps, two genes encoding proteins that condense DNA, did not alter the temporal pattern of gyrB transcription in strains containing deletions in either of these genes. Rather a repressor that specifically recognizes sequences in the gyrB promoter region that are also probably essential for transcription, is likely to be responsible for controlling cell cycle expression.
\end{abstract}




\section{Background}

The bacterium Caulobacter crescentus divides asymmetrically once during each cell cycle, yielding a sessile stalked cell and a motile swarmer cell $[1,2]$. These two cell types differ not only in morphology but also in their ability to replicate their genomes and to divide. The stalked cell immediately reinitiates DNA replication and cell division. The swarmer cell, in contrast, is incompetent for these two processes until it sheds its single, polar flagellum and differentiates into a stalked cell. The stalked cell then passes through several predivisional stages, during which time the genome is replicated and segregated, the newly synthesized DNA is methylated, and a new flagellum is synthesized and constructed at the pole opposite the stalk [14]]. The asymmetry that distinguishes the two daughter cells is founded in the predivisional cell before cell separation occurs. Asymmetric cell division depends upon several general mechanisms, including intracellular protein and/or mRNA localization, regulated phosphorylation and proteolysis, and cell-cycle dependent transcription and translation.

The inability of the Caulobacter swarmer cell to replicate DNA is due in part to the activity of the response regulator CtrA, which binds to sites in the origin of replication (Cori) [5,6]. CtrA is synthesized and phosphorylated when DNA replication is approximately half-way complete; the phosphorylated protein persists in the swarmer cell after cell division $[7,8]$. Phosphorylated CtrA represses transcription of DNA adjacent to the Cori, that is necessary for replication initiation, and also prevents binding of the DnaA initiator protein $[6,9,10]$. When the swarmer cell differentiates into a stalked cell, CtrA is proteolyzed and DNA replication commences [7]. In addition to its role in preventing DNA replication, CtrA is also a transcriptional activator of many genes that affect several different aspects of Caulobacter development. CtrA regulates transcription of its own gene, activates early genes in flagellar biogenesis, and represses $f t s Z$, the earliest known gene in cell division $[5,8,11]$. Interestingly, while microarray analysis has implicated CtrA in the control of approximately 25\% of the cell-cycle regulated genes and it appears to directly regulate 55 operons, it does not regulate the transcription of several genes involved in DNA replication [12,13]. Thus, control of DNA replication initiation also depends on CtrA-independent transcription regulation.

Transcription of many DNA replication genes begins just before DNA replication initiates at the swarmer-to-stalked cell transition. These swarmer-cell-silenced genes include $d n a A, d n a N$ (encoding the $\beta$ subunit of DNA polymerase III), $\operatorname{dnaX}$ ( $\gamma$ and $\tau$ subunits of DNA polymerase III), dnaK (replication-initiation chaperone), and $\operatorname{grB}$ (B subunit of DNA gyrase) [14-18]]. The predicted promoter sequences for most of these genes align well with the published con- sensus sequence for $\sigma^{73}$ [19]. As the promoters of these DNA replication-associated genes appear to contain neither a CtrA binding site or any obvious elements that would suggest transcription activation in stalked cells, the mechanism responsible for their cell-cycle dependent regulation remains unknown.

In this study we analyze the role of the gyrB promoter in swarmer cell-specific silencing of gene expression. We map the site at which transcription of gyrB starts and create deletions to further delineate the promoter sequences required for temporal regulation of the gene to an $80 \mathrm{bp}$ region. Using site-directed mutagenesis we further show that transcriptional silencing in swarmer cells does not appear to depend upon the overall strength of the promoter activity. In vitro experiments demonstrate that gyrB transcription does not require any auxiliary factors, suggesting that temporal regulation is not dependent upon an activator protein. However, gel-mobility shift assays demonstrate that an activity, present in C. crescentus crude whole-cell extracts and unrecognized by anti-RNAP antibody, is able to bind to the gyrB promoter. One possibility is that swarmer-specific silencing is linked to the observed difference in the physical characteristics between the swarmer cell nucleoids and stalked cell nucleoids [20-23]. Thus, a protein that affects the overall folding and structure of the nucleoid might also be regulating transcription. In this regard, we also assayed the effect on gyrB expression in strains containing mutations in either smc or $d p s$, two genes encoding proteins that condense DNA. However we found there was no change in the temporal pattern of gyrB transcription in strains containing deletions in either of these genes.

\section{Results \\ Mapping and deletion analysis of the gyrB promoter}

Previous studies of the gyrB gene found that transcription is silenced in swarmer cells and begins when the swarmer cell differentiates into a stalked cell [17]. These experiments also demonstrated that $g y r B$ is transcribed from its own promoter and is not part of an operon, this despite the fact that the predicted start codon overlaps with the upstream $r e c F$ stop codon [17]. Because the original gyrBpromoterless lac $Z$ reporter fusion used in these studies contained a relatively large region of DNA upstream of the predicted start of translation, we wished to map the gyrB promoter to the minimal region capable of sustaining wild-type temporal regulation of the gene. Primer extension revealed two adjacent transcription start sites, separated from the translation start site by a relatively long leader sequence of approximately 170 base pairs (Fig. 1A). Note that in a separate study a slightly different start site, four base pairs further upstream, was also identified [14]. While we did not detect this start site in either the primer extension assay or in an in vitro transcription/primer 


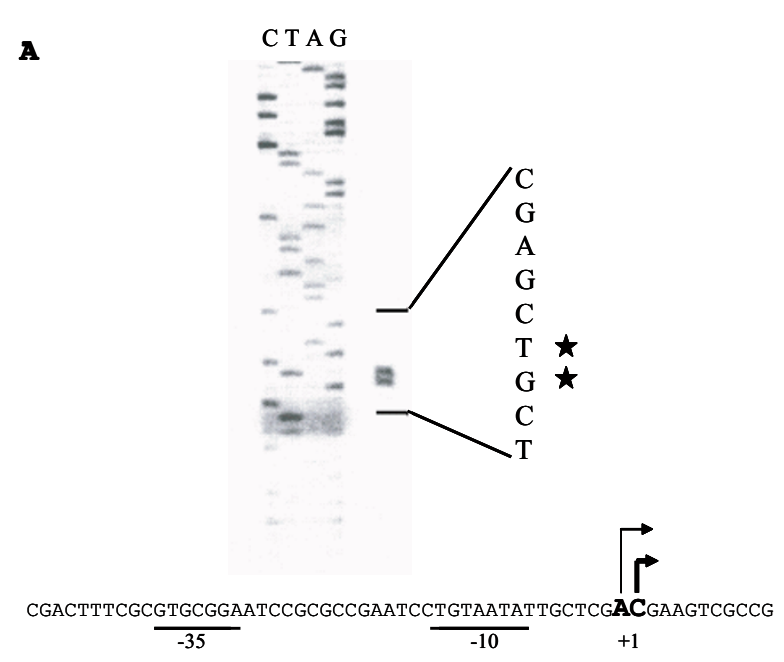

B

$$
\begin{aligned}
& \text { gyrB CGACTTTCGCGTGCGGA ATCCGCGCCGAAT } \frac{-35}{\frac{-10}{+1}}+{ }^{+10} \\
& \text { dnaN TTTCGGCCTCTTCCCCG CGCGCGTCTTTTC GCTAATGTCGGCG GT }
\end{aligned}
$$

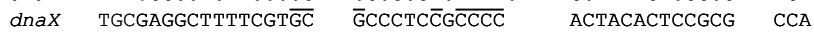

$$
\begin{aligned}
& \text { dnaA AGTTTTCCGTTTGACCG GCCCCCTCCGCTG GCTAGTTTAAGGG } \\
& \text { dnaA AGTTTTCCGTTTGACCG GCCCCCTCCGCTG GCTAGTTTAAGG TC }
\end{aligned}
$$

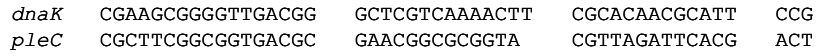

$$
\begin{aligned}
& \begin{array}{lll}
\text { Consensus } & \text { TTGaCgS }
\end{array}
\end{aligned}
$$

\section{Figure I}

The gyrB transcription start site and promoter. (A) The transcriptional start site of gyrB was mapped by primer extension analysis, using an oligonucleotide primer complementary to a sequence just upstream of the translational start site. Transcription initiates at two adjacent sites, indicated by stars, approximately 170 bp upstream of the predicted start of translation. (B) The gyrB promoter was compared with other known $\sigma^{73}$ promoters, both cell-cycle regulated and constitutive; predominantly the former are shown [14-16,19,24-26]. The start sites and predicted - 10 and -35 promoter elements are indicated above the sequence alignment; the published $C$. crescentus consensus sequence for genes transcribed by the common $\sigma^{73}$ RNAP holoenzyme is shown below, where $\mathrm{S}$ stands for $\mathrm{G}$ or $\mathrm{C}, \mathrm{W}$ indicates $\mathrm{A}$ or $\mathrm{T}$, and $\mathrm{N}$ means any nucleotide $[19,26]$. The lines above the dnaX sequence indicate the previuosly identified RRF element [24].

extension experiment (see below), the alternative transcription start site was taken into consideration in subsequent mutagenesis experiments.

Based on the transcription start sites found by primer extension, alignment and comparison with several other known $\sigma^{73}$ genes allowed identification of possible -10 and -35 promoter elements of gyrB (Fig. 1B) [14-16,19,24-
26]. The consensus is derived from $\sigma^{73}$ transcribed genes that are expressed at relatively constant levels throughout the cell cycle; expression of gyrB, along with other DNAreplication genes, varies during the cell cycle. In analyzing the gyrB promoter and surrounding DNA, we considered two broad possibilities for gyrB transcription regulation. gyrB expression might be controlled by the activity of a swarmer cell-specific factor that inhibits transcription or by a stalked cell-specific factor that activates transcription. An (A+T)-rich region, beginning approximately $60 \mathrm{bp}$ upstream of the transcription start site, at first appeared to be a good candidate for a possible regulatory protein binding site. This region consists of two stretches of repeated thymidine residues and one stretch of repeated adenine residues, approximately phased with respect to each other on the DNA strand (data not shown). Such an arrangement could create a bend in the DNA, a secondary structure that is preferred by some DNA-binding proteins and that can affect transcription independent of any additional protein constituents $[27,28]$. Another part of the gyrB gene that might possibly contain a cis-acting regulatory site is the extremely long (170 bp), untranslated leader sequence between the transcription and translation start sites. Alternatively, gyrB transcription might not depend on any remote DNA elements or sites for sequence-dependent DNA binding proteins. As has been reported, the putative gyrB promoter appears more similar to a subset of $\sigma^{73}$ genes that mirror its temporal regulation than to those genes that are constitutively expressed throughout the cell cycle (Fig. 1B) $[14,17,24,26]$. This would seem to suggest that temporal regulation is dictated by the promoter itself rather than by a more distantly located DNA element.

In order to begin dissection of the gyrB promoter, we used PCR to create deletions both upstream and downstream of the core $\sigma^{73}$ promoter. We measured the transcriptional activity from these shortened promoters, each fused to a promoter-less lac $Z$ gene, by $\beta$-galactosidase activity. Deletion of the upstream sequences, including the $(\mathrm{A}+\mathrm{T})$-rich region, resulted in a slight, but not statistically significant, decrease in gyrB transcription (pJEZP2 and pJEZP3) (Fig. 2). A similar decrease was seen when approximately 100 bp of the untranslated leader sequence was deleted (pJEZP2s). However, wild-type transcription levels were restored by combined removal of the leader and upstream sequences (pJEZP3s, pJEZP4) and/or by removal of an additional 49 bp of the leader sequence (pJEZP5). These results indicate that the overall strength of gyrB promoter activity depends upon at most an 80 bp promoter region, covering -53 to +27 bp relative to the start of transcription. The temporal regulation of the deleted promoters was then investigated by immunoprecipitation of $\beta$-galactosidase produced from each construct during growth in synchronized cultures. The expression pattern of the deleted 


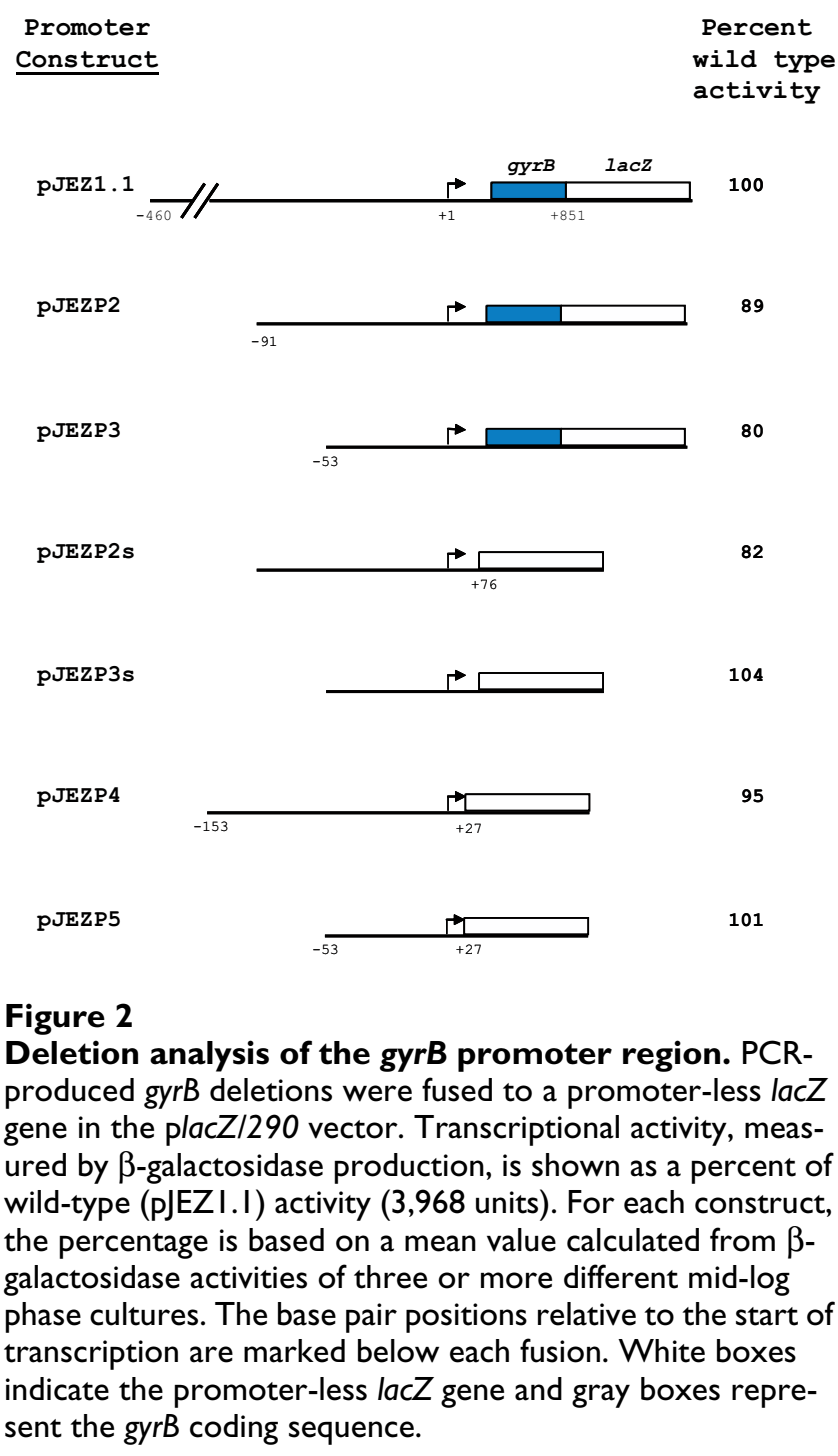

promoter fusions during the cell cycle was similar to that observed for the full-length fusion (Fig. 3). In all cases, transcription was barely detectable in swarmer cells $(0$ division units) followed by a marked increase in transcription at the swarmer-to-stalked cell transition (0.2 division units) and then a subsequent decrease in late predivisional cells (0.8 division units) (Fig. 3 ). Thus, the temporal control of the gyrB gene is also dictated by the core, $80 \mathrm{bp}$ promoter.

\section{Mutagenesis of the gyrB promoter}

One attractive idea to explain the temporal regulation of gyrB transcription is that a deviation in promoter sequences from that of the $\sigma^{73}$ consensus results in a

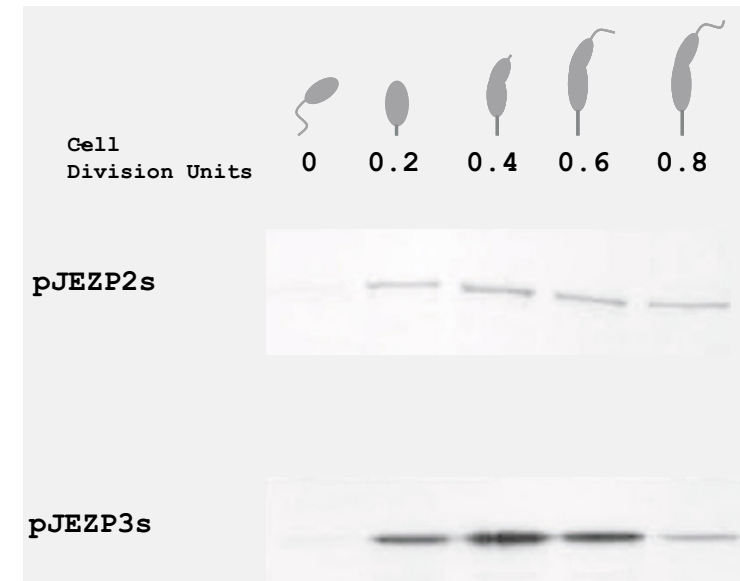

PJEZP4

PJEZP5

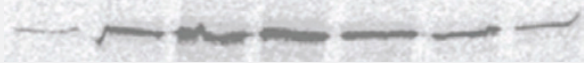

Figure 3

Temporal expression patterns of the gyrB promoter fusions during the $C$. crescentus cell cycle. Transcription of the gyrB-lac $Z$ fusions contained on the indicated plasmids was examined during synchronized growth by immunoprecipitation of pulse-labeled $\beta$-galactosidase, as described in Material and Methods. The lanes, each revealing the $\beta$-galactosidase synthesized during one five-minute interval, are aligned beneath a diagram showing the corresponding stage in the cell cycle, as determined by light microscopy. 'Cell division units' refer to fractions of one complete cell cycle, the duration of which is approximately 140 minutes under the experimental conditions employed here. Samples were labeled every 30 minutes or every 20 minutes (pJEZP5).

expression levels that are somewhat weaker than that of constitutively expressed promoters. This property might make gyrB transcription more susceptible to a general silencing mechanism present in swarmer cells, whereas stronger $\sigma^{73}$ promoters would be expressed at normal levels. We investigated the importance of the basic architectural elements of the gyrB promoter, relative to the consensus sequence derived from constitutive $\sigma^{73}$ promoters, by site-directed mutagenesis (Fig. 4). The mutations were designed to bring the gyrB promoter into better agreement with the $C$. crescentus $\sigma^{73}$ consensus $[19,26]$. 
A

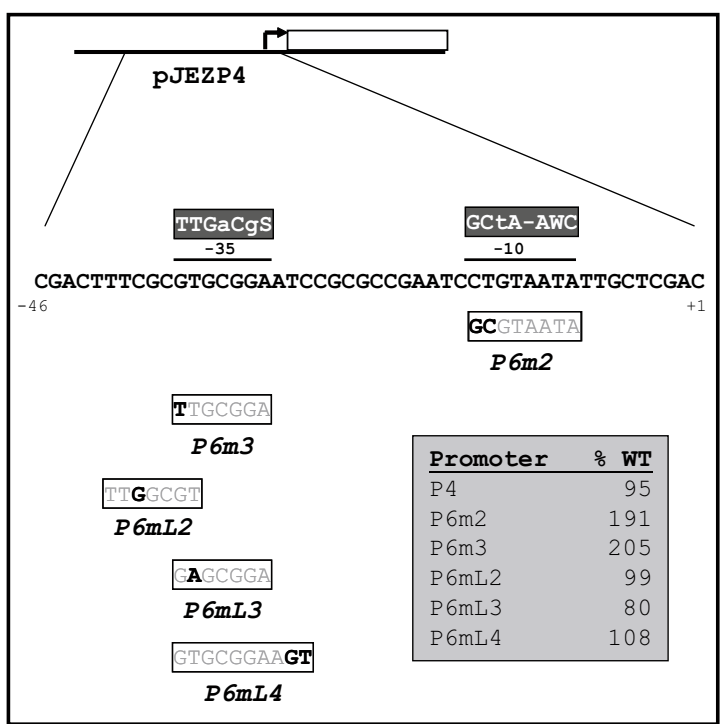

B

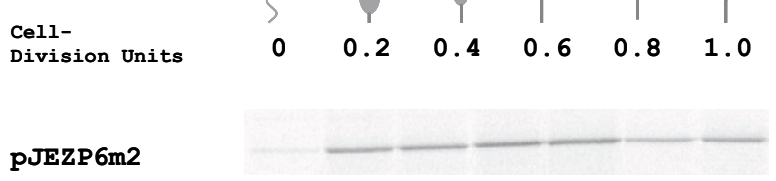

PJEZP6m3

\section{Figure 4}

Transcription of gyrB promoter mutations designed to conform to the $C$. crescentus $\sigma^{73}$ promoter consensus. (A) The plasmid pJEZP4 contains the template promoter construct used for mutagenesis (see Fig. 3). Shown below is the DNA sequence of the promoter, with the -10 and -35 consensus regions in reverse highlight above. The mutations, shown as boxed sequences with the altered bases in bold-face, were based upon sequence alignment and were aimed at increasing the promoter's conformity with the accepted $\sigma^{73}$ consensus sequence. In a separate study, an alternative start site and subsequent alignment located the gyrB promoter further upstream; the mutations designed to take these data into account include an "L" in their names [14]. The transcriptional activity from the mutant promoters, as determined by $\beta$-galactosidase measurements of the corresponding lac $Z$ fusions, is presented in the box in the lower right-hand corner as a percentage of wild-type (pJEZI.I). (B) Temporal expression patterns of the gyrB promoter mutants. Transcription from the gyrB promoter mutants was analyzed during the $C$. crescentus cell cycle. The results for the mutants exhibiting the strongest quantitative transcription levels (pJEZP6m2 and PJEZP6m3) are shown beneath a schematic of the cell cycle that shows the corresponding developmental stage of the synchronized culture. Samples were labeled every 20 minutes.
The P6m 2 mutation changed the bases CT at -16 to -15 to GC, conserved at those positions in the consensus; likewise, $\mathrm{P} 6 \mathrm{~m} 3$ created a $\mathrm{T}$ at the -37 position. Because an alternate start site and promoter were published while these experiments were in progress, we also designed mutations based on these findings, so designated by the letter " $\mathrm{L}$ " in the name of the mutation [14]. The mutations P6mL2 and P6mL3 were aimed at increasing the consensus agreement of the -35 region published previously $[19,26] ;$ P6mL4 targeted a proposed 13-mer motif located between the -10 and -35 region that was also previously identified [24].

Each mutated promoter was fused to a promoterless lacZ gene and expression levels were quantitatively measured by $\beta$-galactosidase assays (Fig. $4 \mathrm{~A}$ ). The mutations $\mathrm{P} 6 \mathrm{~m} 2$ and $\mathrm{P} 6 \mathrm{~m} 3$ resulted in transcription levels that were twice that of the wild-type level, while the P6mL2, -3 , and -4 were transcribed at normal or (P6mL3) reduced amounts. The increased promoter activity of $\mathrm{P} 6 \mathrm{~m} 2$ and $\mathrm{P} 6 \mathrm{~m} 3$ and the decreased expression of P6mL3 support the proposed promoter sequence reported here (Fig. 1) as the P6m2 and $\mathrm{P} 6 \mathrm{~m} 3$ mutations increase the agreement with the $\sigma^{73}$ consensus and while the P6mL3 mutation decreases the conformity. One possibility that could account for the increased promoter activity of the $\mathrm{P} 6 \mathrm{~m} 2$ and $\mathrm{P} 6 \mathrm{~m} 3$ mutant promoters was inappropriate expression in swarmer cells. Therefore, we wished to examine the temporal transcription patterns of these promoters in order to test this possibility (Fig. 4B). Surprisingly, transcription from the mutant promoters, though stronger, was still regulated in the wild-type manner during the cell cycle (Fig. 4B).

\section{Transcription of gyrB does not require an activator}

Because simply creating a stronger $\sigma^{73}$ promoter failed to have any effect on the temporal pattern of gyrB transcription, we wished to rule out the possibility that gyrB requires a transcriptional activator for expression within stalked cells. We employed an in vitro transcription assay to address this issue, using a commercially obtained preparation of E. coli $\left(\sigma^{70}\right)$ RNA polymerase (RNAP). In this experiment, transcription from a promoter on a supercoiled plasmid was assayed by primer extension. Transcription products were detected using both preparations of RNAP, indicating the lack of a requirement for an auxiliary transcriptional activation factor (Fig. 5). Of the several start sites observed, one site was present when either RNAP species was used in the in vitro assay (Fig. 5) as well as when RNA was obtained directly from cultured cells (see Fig. 1A). The presence of one common start site is further evidence for the correct identification of the gyrB promoter reported here. From this experimental result, we can conclude that the sole protein requirement for in vitro 


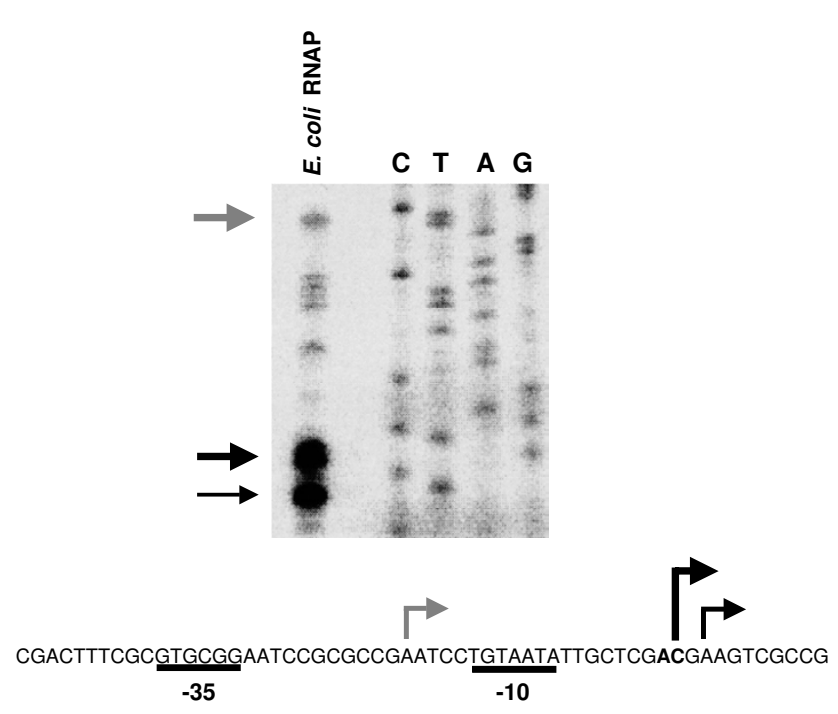

Figure 5

In vitro transcription/primer extension of gyrB. In vitro transcription, followed by primer extension, was performed on the gyrB promoter template pJEKS-P3. A $\sigma^{70}$ RNAP holoenzyme preparation, obtained from $E$. coli $\sigma^{70}$ was used for transcription assays. Shown at the top are the DNA species, produced from the extended messages, next to a sequencing ladder generated from the same primer. Strong start sites are indicated by larger arrows, weaker start sites by smaller arrows. Below, is the complimentary sequence of the gyrB promoter, with the -10 and -35 promoter elements indicated. The major transcription start sites found by this assay are shown by upward-facing arrows, while the adjacent start sites previously found by primer extension (see Fig. I) are shown in bold; the largest upward-facing arrow indicates a start site appearing in both assays and with both RNA polymerase species.

transcription of the $g y r B$ gene is the $\sigma^{70}\left(\sigma^{73}\right)$ RNAP holoenzyme.

\section{An activity, distinct from RNA polymerase, binds the gyrB promoter in vitro}

Because transcription from the gyrB promoter did not appear to require an activator protein, it seemed likely to us that temporal regulation of the gene during the cell cycle was not attributable to stalked cell-specific transcriptional activation. An alternative possibility is that the gene is specifically repressed in swarmer cells and in the swarmer pole of the predivisional cell, possibly by a protein that binds to the promoter. We therefore decided to use gel-mobility shift assays to look for any binding activities present in C. crescentus crude cell extracts that interacted with the gyrB promoter. A very weak shift was observed when fresh crude extract was incubated with the probe (Fig. 6A, lanes 2-3, white broken arrow); this shift is apparently due to the binding of RNAP, evidenced by the further retardation of mobility when anti-RNAP antibody was added to the incubation mixture (Fig. 6A, lanes 5-7). An additional complex was seen with increasing volumes of crude extract (Fig. 6A, lanes 3-4, gray arrow). This binding activity appears to be distinct from RNAP binding as the shifted band is unaffected by the addition of anti-RNAP antibody (Fig. 6A, lanes 5-7).

Interestingly, we observed different results when we compared samples containing fresh extracts (and extracts that had been frozen only once) with those containing extracts that had been previously thawed and then refrozen. The non-RNAP binding activity was labile under conditions in which the extract had been subjected to one round of freeze-thaw (Fig. 6B, lanes 2 and 3 vs. lanes 4 and 5). Furthermore, when the extract that had been refrozen and thawed was used in the binding assay (Fig. 6B, lanes 2,3), the disappearance of the specific, labile protein band was accompanied by the appearance a new specific supershifted band upon the addition of anti-RNAP antibody.

\section{The role of DNA condensing proteins in the regulation of gyrB transcription}

Previous studies have shown that, in addition to being unable to initiate DNA replication, C. crescentus swarmer cells also differ from stalked cells in their chromosomal architecture [21-23]. These experiments revealed that isolated swarmer cell nucleoids sediment faster in a sucrose gradient than those of stalked cells. One hypothesis is that the swarmer cell nucleoid possesses a different complement of DNA 'condensing' proteins such as histone-like proteins. The gel mobility shift activity present in crude cell extracts may reflect the binding of a protein that alters the overall architecture of the swarmer cell nucleoid. Two candidates for such an activity may be either Dps or SMC. In E. coli, the Dps protein is induced both in stationary phase and during starvation; it is able to physically protect the nucleoid from oxidative and nuclease damage, as well as affect transcription under these conditions [29-32]. SMC ( Structural Maintenance of Chromosomes), is a member of the eukaryotic SMC family [reviewed in [33-36]], large, multidomain proteins that associate with each other in an antiparallel fashion. This association occurs through long, coiled-coil domains that flank a central hinge region, resulting in protein dimers that are shaped like old-fashioned hairpins. Dimers of SMC associate with non-SMC factors to form specific complexes in eukaryotic cells, with functions in DNA organization and movement, ranging from condensation to dosage compensation to repair [33,37]. Experiments in Bacillus subtilis have demonstrated that chromosomal DNA was visibly decondensed in smc mutants [38]. 
A
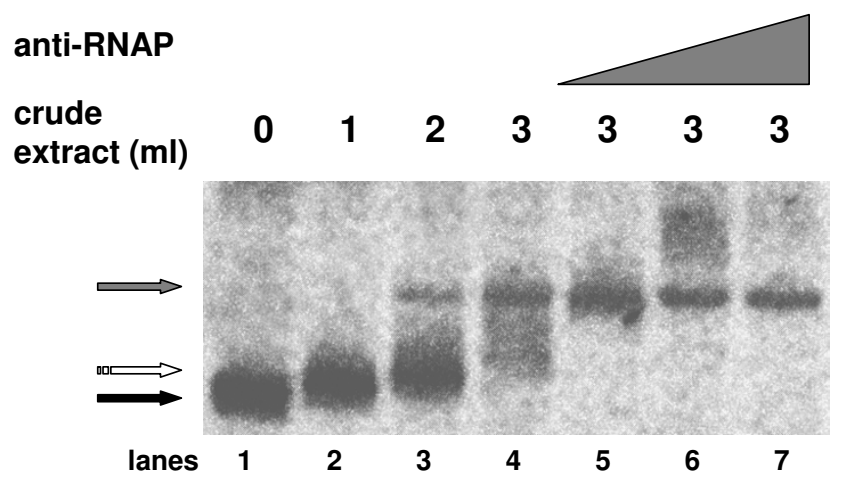

B

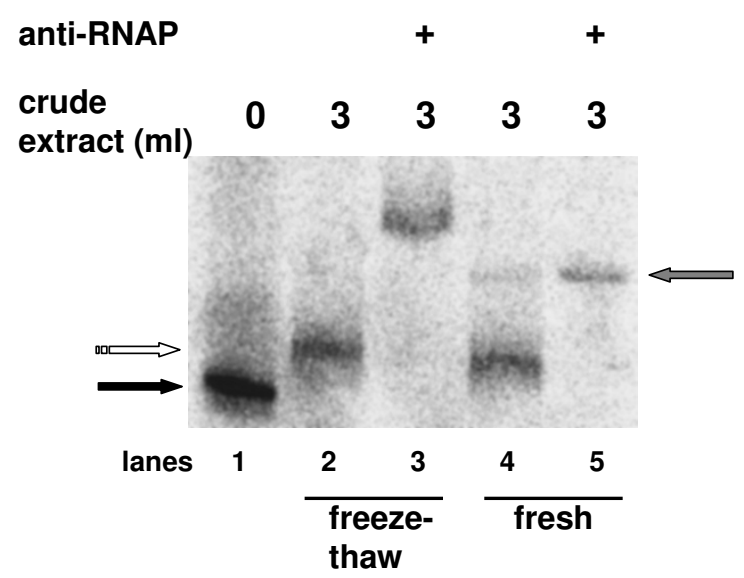

Figure 6

Gel-mobility shift assays reveal a distinct, labile activity that binds to the gyrB promoter. (A) A 500 base pair [32P]-end-labeled DNA fragment containing the gyrB promoter was used as a binding template for incubation with increasing amounts of fresh C. crescentus crude cell extract (approximately I to $3 \mathrm{mg} / \mathrm{ml}$ ), without (lanes I-4) or with (lanes 5-7) increasing amounts of anti-RNAP antibody. (B) The same binding template was incubated with $3 \mu \mathrm{l}$ of fresh crude extract (lanes 4-5) or extract that had been previously re-frozen (lanes 2-3), with and without anti-RNAP antibody $(3 \mu \mathrm{l})$. All binding mixtures were incubated at room temperature for 10 minutes and immediately loaded onto native $6 \%$ polyacrylamide gels. Black solid arrows indicate probe alone; gray solid arrows indicate a potential freeze-thaw-labile, shifted species; white, broken arrows indicate a binding activity that is super-shifted by anti-RNAP antibody.

The Caulobacter $d p s$ gene is approximately 50 to $55 \%$ identical and 60 to $65 \%$ similar to $d p s$ genes in other organisms, including Burkholderia pseudomallei, Bordetella pertussis and Pseudomonas syringae [data not shown]. Furthermore, a search of the NCBI CDD (conserved domain database) revealed significant alignment with a COG (cluster of orthologous genes) for Dps/ferritin-like DNAbinding proteins. The gene appears to be the first in a twogene operon; the expected product of the second gene is a conserved hypothetical protein of unknown function. A lacZ transcription fusion of a DNA fragment spanning 600 bp upstream of the predicted $d p s$ translation start site was expressed at a low level (350-400 units of $\beta$-galactosidase activity) in late log-phase cultures [data not shown]. Although it has been shown to be involved in DNA protection and starvation and stationary-phase survival in other organisms, it seemed possible that Dps might be involved in developmental events specific to the swarmer cell: the results of genome-wide DNA microarray experiments showed that $d p s$ mRNA is maximally present in swarmer cells $[13,30]$. As one way to investigate a possible role for Dps in Caulobacter development, we engineered a strain in which $90 \%$ of the $d p$ s coding sequence was replaced with a gene conferring resistance to gentamicin. The strain was indistinguishable from wild-type cells in logarithmic growth and morphology, and we detected no overt aberration in swarmer-cell motility or nucleoid appearance (data not shown). Additionally, we constructed a strain with a null mutation in $s m c$ by disrupting the chromosomal copy of the $s m c$ gene by insertion of a gene conferring spectinomycin resistance [see Methods]. The deletion was confirmed by Southern blot and by immunoblot with antibodies against SMC [data not shown].

Finally, we examined the cell-cycle transcription pattern of gyrB in the smc and $d p s$ mutant strains. The temporal expression pattern of a gyrB fused to a promoter-less $l a c Z$ gene was assayed by immunoprecipitation of pulselabeled $\beta$-galactosidase in synchronized cultures of NA1000 (wild-type), JG3003 and JG3402 (Fig. 7). In both mutant strains, the lac $Z$ gene was transcribed in a manner comparable to the transcription observed when the same promoter fusion was expressed in NA1000. Thus, SMC and Dps do not appear to participate in swarmer-specific transcription silencing in Caulobacter crescentus.

\section{Discussion}

In Caulobacter crescentus, many of the genes involved in DNA replication, including $d n a N, d n a X, d n a A, d n a C, d n a K$ and $\operatorname{gyr} B$, are maximally expressed at the time that this process occurs [14-18,24,25]. Thus, transcription of these genes does not occur in swarmer cells, or in the swarmer pole of predivisional cells, and begins only when the swarmer cells differentiate into stalked cells or when DNA replication commences anew in the stalked cells at the completion of cell division. The means whereby this regulatory pattern is achieved remains unknown. In this 

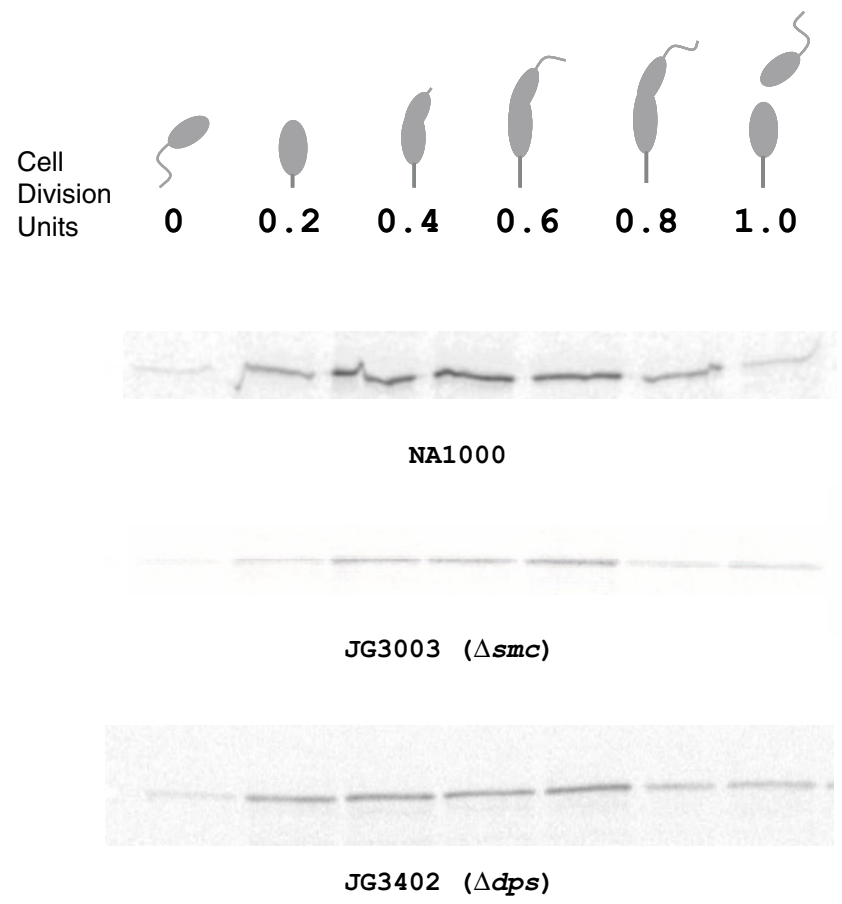

\section{Figure 7}

The gyrB promoter is expressed in the wild-type temporal pattern in smc and $d p s$ null strains. The plasmid PJEZP5, containing the gyrB promoter fused to a promoterless copy of lacZ, was moved into NAI000 (wt, top), JG3003 (middle) and JG3402 (bottom) by conjugation. Transcription from the gyrB promoter was monitored during synchronized growth of the two strains by immunoprecipitation of $\beta$-galactosidase pulse-labeled at intervals during the cell cycle. For each strain, the protein labeled during each five-minute interval is shown beneath a diagram of the corresponding stage in the cell cycle, as determined by light microscopy.

study, we used gyrB as a representative gene with which to investigate the mechanism responsible for swarmer-specific transcription silencing in C. crescentus. As revealed by primer extension and in vitro transcription/primer extension assays, the transcription start site of the gene is separated from the translation start site by a fairly long leader sequence. There is apparent variation in the precise beginning of the transcript, as observed here and as reported elsewhere [14]. The lack of a precise transcription start site may be due to other factors that influence gyrB regulation. For example, transcription of the gyrB gene in Caulobacter is induced by relaxation of the DNA [17], and this induction is distinct from the increased expression that occurs during the swarmer-to-stalked cell transition. It is also possible that the transcription start site varies depending upon undefined regulatory cues such as growth rate and/ or growth phase of the culture.
Having delineated the gyrB promoter to a minimal, 80 base pair region, we compared this promoter to other $\sigma^{73}$ promoters and to the published consensus sequence, taking care to explore different alignments based on the several possible start sites. The alignment shown [see Fig. 1] is that best fitting the major start site obtained by both primer extension and in vitro transcription/primer extension (for an alternative, see [14]). Examination of the -10 and -35 regions of the promoter, based upon our alignment, revealed that the gyrB promoter greatly differs from the $C$. crescentus $\sigma^{73}$ consensus sequence. This suggested an interesting possibility for a swarmer-specific silencing mechanism. We reasoned that greater deviation from the consensus sequence might make these promoters slightly inferior binding sites for the $\sigma^{73}$ RNAP, and thus slightly weaker. Such promoters might be more susceptible to a situation in which RNAP binding is partially inhibited or blocked, a possible scenario occurring in swarmer cells. Site-directed mutagenesis allowed us to test this possibility by creating versions of the gyrB promoter that more closely matched the consensus sequence and therefore should, in theory at least, have been transcribed at higher levels. However, while in two cases transcription from the mutated promoters was greater than from the wild-type promoter, temporal regulation of transcription was unaffected. Thus, it seems that the strength of the promoter does not intrinsically determine cell cycle regulation of gyrB.

The experiments presented here show that the gyrB promoter can be transcribed by $E$. coli $\sigma^{70}$ RNA polymerase holoenzyme in the absence of additional protein factors, suggesting the unlikelihood of temporal regulation depending solely upon an activator. However, it is possible that a stalked-cell specific transcriptional activator is required to overcome a swarmer-cell specific repression complex or DNA topological conformation. When a probe containing the gyrB promoter was used in gelmobility shift assays, two binding activities, both of which are components of crude Caulobacter extracts, were apparent. One of these activities was loosely identified as RNA polymerase based on the ability of anti-RNAP antibody to recognize and alter the migration of this band through the gel. The other binding activity was not recognized by the antibody. This second species was vulnerable to freezethaw treatment, as it was only present in extract that had not been re-frozen. One might imagine a scenario in which a swarmer-specific DNA protein(s) binds to the gyrB promoter and interferes with the ability of the polymerase to access the DNA.

Previous experiments with another swarmer cell-repressed promoter, the dnaX promoter, revealed the RRF motif, that by sequence comparison is located between the -10 and -35 elements in all of the swarmer cell-silenced DNA 
replication gene promoters [24]. Within the RRF of the dnaX promoter, a $\mathrm{C}$ residue at position -21 appears to be involved in the temporal regulation of the gene [24]. A substitution of $\mathrm{T}$ at this position results in a more than 3fold increase in transcription and, more importantly, in a loss of swarmer-specific silencing of $d n a X$. Additionally it was shown that the RRF motif is bound by an unknown species found in crude extracts [24]. The same shift was seen when DNA fragments encompassing the - 10 and -35 regions of several different DNA-replication genes, including gyrB and $d n a X$, were used as probes in gel-mobility shift assays. It is possible that the binding activity reported here for the gyrB promoter is the same as that observed previously with the RRF motif. If this observed binding activity were responsible for the swarmer cell-specific silencing of gyrB transcription, the one might expect that swarmer cell extracts would be enriched in binding activity. However, we were unable to detect this activity in extracts derived from either isolated swarmer or stalked cells, probably owing to limitations in obtaining a sufficient quantity of material. The relevance of these DNA binding activities and a experimental definition of their binding sequences in similarly regulated promoters will need to be explored.

The condensed nature of nucleoids isolated from swarmer cells, relative to stalked cells, when subjected to centrifugation through a sucrose gradient, suggests that the two cell types differ in the proteins that affect the organization and folding of their chromosomes [20]. Growth-phase dependent variation in the relative composition of the DNA-binding protein cadre in E. coli has been documented [39]. A similar developmentally-regulated phenomenon might be responsible for silencing gyrB and other genes involved in DNA replication in Caulobacter swarmer cells. Proteins such as HU or H-NS bind DNA nonspecifically and have been shown to affect transcription regulation [40]. While neither protein binds to a specific sequence on the DNA, each has a preference for certain secondary DNA structures, including gaps and junctions (HU) and (A+T)-rich bends (H-NS). Of particular interest, H-NS represses the virF gene by binding to two sites, one overlapping the RNAP binding site in the promoter and another 200 bp upstream [41]. However, repression only occurs at temperatures below $32^{\circ} \mathrm{C}$ because below this temperature the DNA is more intrinsically bent, which allows interaction between H-NS bound at both sites. HU, the most abundant DNA-binding protein in bacteria, also regulates transcription. Similar to integration host factor, $\mathrm{HU}$ is thought to exert its influence by creating a bend in the DNA and thus affecting the activity of other, more specific transcription regulators [reviewed in $[40,42,43]]$. Based on searches of the published genome, we have concluded that $C$. crescentus does not possess a gene that might encode H-NS, nor does it appear to have a homolog of the closely related gene, stpA [42]. C. crescentus, does however, possess genes encoding proteins that are closely homologous to the two subunits

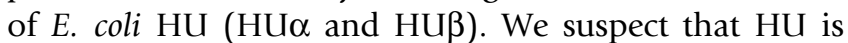
probably not involved in the regulation of the DNA replication genes since we have recently found that the abundance of both HU subunits does not change during the course of the cell cycle [unpublished observation].

In order to determine whether or not two other wellknown DNA condensation proteins might affect swarmerspecific transcription, we constructed strains in which $s m c$ or $d p s$ were disrupted. There is strong evidence that the large bacterial SMC protein, very different from the small DNA-binding proteins discussed above, is involved in chromosome condensation and partitioning, similar to its eukaryotic homologs $[33,36]$. A deletion of the gene encoding SMC in Bacillus subtilis results in a temperaturesensitive strain with visibly decondensed chromosomes and defects in chromosome segregation $[44,45]$. Similar results have also been reported in Caulobacter crescentus [46]. Importantly, however, temporal transcription of gyrB was unchanged in the $\Delta s m c$ mutant, indicating that SMC is not necessary for swarmer-specific transcriptional silencing.

The final target of our studies was the Dps protein. in $E$. coli, Dps protects DNA during periods of starvation or in stationary phase $[31,32]$. When bound to DNA, Dps forms a crystalline structure that is proposed to render the DNA physically resistant to oxidative damage as well as degradation by some nucleases [29]. Dps is also important for induced DNA protection during non-stationary phase growth, as dps mutants are more sensitive to hydrogen peroxide [47]. The nature of the Caulobacter swarmer cell (DNA replication- and cell division-incompetent) makes it conceivable that the intracellular environment may be likened to that of a cell in stationary phase or starved for nutrients. Thus, perhaps Dps plays a different role in Caulobacter. Supporting this hypothesis, DNA microarray experiments have revealed that the $d p s$ mRNA level is greatest in swarmer cells and then decreases dramatically at the swarmer-to-stalked cell transition [13]. A deletion of $d p s$, however, did not have a visible effect on the Caulobacter cell cycle. Also, similar to the $\Delta s m c$ strains, the temporal expression pattern of the gyr $B$ promoter was unaffected in the $\Delta d p s$ strain.

\section{Conclusion}

In summary, these results show that a core 80 bp gyrB promoter sequence is sufficient to confer a cell cycle-regulated pattern of transcription. In vitro assays indicated that $\mathrm{gyrB}$ transcription does not require any factors in addition to RNAP. Thus, the transcription of gyrB is likely to be regulated by a repressor protein in swarmer cells, and not an 
Table I: Strains and Plasmids

\begin{tabular}{|c|c|c|}
\hline Strain & & Reference \\
\hline \multicolumn{3}{|l|}{ E. coli } \\
\hline SI7-I & $R p 4-2, T c:: M u, K m:: T n 7$ & [49] \\
\hline TG-I & $\mathrm{F}_{-}$, lacla, proA ${ }^{+} B^{+}$lacZ_MI5 & [6I] \\
\hline \multicolumn{3}{|l|}{ C. crescentus } \\
\hline NAI000 syn-I000 (synchronizable strain) & & {$[22]$} \\
\hline JG3003 syn-1000, smc7 (spec resistance) & & [this study] \\
\hline JG3402 syn-1000, dps2 (gent resistance) & & [this study] \\
\hline Plasmids & & Reference \\
\hline pBluescript-KS ${ }^{+}$ & cloning vector & [Stratagene] \\
\hline placZ/290 & pRK290 derivative containing a promoterless lac $Z$ gene & [48] \\
\hline pNPTSI39 & sacB counter selection vectors; $\left(k^{r}{ }^{r}\right)$ & [M.R.K. Alley] \\
\hline PJEKS- gyrB500 & $500 \mathrm{bp}$, Pstl/Stul gyrB fragment in pBluescript-KS ${ }^{+}$ & [this study] \\
\hline PJEKS-P3 & 904 bp Pstl/Hindlll gyrB promoter fragment & [this study] \\
\hline PJGZI.65 & I.65 kb Pstl/EcoRI fragment in placZ/290, gyrB-lacZ transcriptional reporter fusion & {$[17]$} \\
\hline PJEZI.I & I.3 kb Pstl/HindIII fragment in placZ/290 & [this study] \\
\hline PJEZP2 & 942 bp Pstl/HindIII gyrB-lacZ transcriptional reporter fusion & [this study] \\
\hline PJEZP3 & 904 bp Pstl/HindIII gyrB-lacZ transcriptional reporter fusion & [this study] \\
\hline PJEZP2s & 167 bp Pstl/(native)Stul gyrB-lacZ transcriptional reporter fusion & [this study] \\
\hline PJEZP3s & I 32 bp Pstl/(native)Stul gyrB-lacZ transcriptional reporter fusion & [this study] \\
\hline PJEZP4 & I80 bp Pstl//HindIII gyrB-lacZ transcriptional reporter fusion & [this study] \\
\hline PJEZP5 & 80 bp Pstl/Hindlll gyrB-lacZ transcriptional reporter fusion & [this study] \\
\hline
\end{tabular}

activator protein. This repressor may be related to the DNA binding activity present in crude cell extracts that bound specifically to the gyrB promoter region. Deletions in the genes encoding the global DNA binding proteins, SMC and Dps had no effect on viability, growth rate or the temporal transcription pattern of gyrB. Thus, the results of this study do not provide definitive support for the idea that DNA-binding proteins that alter the global architecture of chromosomal DNA are involved in transcriptional repression in the C. crescentus swarmer cell. However, it is possible that the condensation of the swarmer-cell nucleoid is the result of the combined activity of two or more proteins and this may be important in influencing gyrB transcription.

\section{Methods}

Bacterial strains, growth conditions, and plasmid construction

Table 1 shows the strains and plasmids employed in this study. The gyrB fragments used in construction of promoter fusions were created by polymerase chain reaction
(PCR), unless otherwise indicated, with the indicated restriction sites introduced on oligonucleotides otherwise complementary to the wild-type DNA sequence. The exception to this is the plasmid pJEZ1.1, in which the gyrB promoter fragment is derived from an upstream, native PstI site ( $\sim-460$ bp from the transcription start site), and the plasmid pJEKS-gyrB500, in which the 500 bp gyrB fragment is derived from the same native PstI site and a native $S t u$ I site at position +76 bp from the transcription start site. Generally, DNA fragments were first inserted into the plasmid pBluescript-KS+ before subsequent subcloning. The template used for the PCR reactions was purified C. crescentus (NA1000) chromosomal DNA; the base plasmid (placZ/290) [48] containing the fusions is a low-copy-number, plasmid, introduced into Caulobacter from a host $E$. coli strain (S17-1) by conjugation [49]. All E. coli cultures were grown at $37^{\circ} \mathrm{C}$ with aeration in $\mathrm{LB}$ broth containing (if applicable) the appropriate antibiotic [50]. C. crescentus strains were grown with aeration at $31^{\circ} \mathrm{C}$ in either peptone yeast extract (PYE) [51] or M2 minimal medium containing glucose [52]. 
The $d p s$ deletion strain was generated by the $s a c B$ selection method [53]. In order to accomplish this, the dps gene was first isolated by PCR as two separate DNA fragments that were subcloned into pBluescript $\mathrm{KS}+$, reconstituting the gene with a newly-created internal MluI site. The coding region was then disrupted by introducing a gentamicin resistance cassette from pKnock GM [54] into the unique MluI site. This entire fragment, $d p s 2 \Delta g$, was then subcloned into the $s a c B$ selection vector, pNPTS139 [M.R.K. Alley, unpublished]. The plasmid, pNPTSdps $2 \Delta \mathrm{g}$, harbored in E. coli strain S17-1, was introduced into $C$. crescentus strain NA1000 by conjugation. Transconjugants were selected from PYE agar plates containing kanamycin and naladixic acid $(20 \mu \mathrm{g} / \mathrm{ml})$. A $2 \mathrm{ml}$ culture inoculated with several transformants was grown for approximately 8 hours in PYE without selection. The culture was then diluted $1 / 10$ and plated $(0.2 \mathrm{ml} /$ plate) on PYE agar containing $2.5 \%$ sucrose. One to three hundred sucrose resistant colonies were than spotted onto two different growth media: PYE sucrose plates containing gentamicin and PYE plates containing kanamycin. Sucrose-resistant, kanamycin-sensitive, gentamicin-resistant colonies where isolated for further study. The deletion of $d p s$ in strain pJG3402 was confirmed by PCR. The smc deletion strain was also isolated by this basic method except that the smc gene was disrupted with a spectinomycin resistance cassette. The smc deletion was confirmed by Southern blot (data not shown) and by immunoblot with anti-SMC antibodies (not shown). Following allelic exchange, the disrupted $d p s$ and $s m c$ genes were each transduced into a new wild-type C. crescentus strain [53].

\section{Cell cycle experiments}

The transcription of the lacZ reporter fusions was assayed in cultures synchronized essentially as described previously [21]. Isolated swarmer cells were suspended in M2 glucose medium and allowed to continue growth. At various time points, $5 \mathrm{ml}$ portions of growing culture were removed and the proteins were pulse-labeled for $5 \mathrm{~min}$ utes with ${ }^{35}$ S-Trans-label (ICN). Labeled $\beta$-galactosidase was immunoprecipitated as described previously [17].

\section{Site-directed mutagenesis and molecular biology procedures}

The initial template used for mutagenesis was the $180 \mathrm{bp}$ gyrBP4 promoter fragment. The fragment was cloned into M13-BM20 and site-directed mutagenesis was performed on single-stranded DNA isolated from E. coli TG-1, as previously described [55]. The mutant gyrB promoters were confirmed by sequencing performed according to the dideoxy chain-termination method [56]. The $\beta$-galactosidase was assayed as previously described $[57,58]$, and the reported values represented the mean activity from at least three independently grown cultures assayed in triplicate. The standard deviation in all cases was less than 5\%. All other routine molecular biology manipulations were performed as previously described [50].

\section{Primer extension and in vitro transcription/primer extension}

Primer extension, both singly and following in vitro transcription, was performed as previously described [50]. The oligonucleotides (PE1: 5'-GCGTCGCCACGCGAACGC-3'; P E 2 : 5 ' - T G A G T T C G T C A G C C A G A G C - 3 ' ; B S 2 : 5 ' -GAGCAATATTACAGGATTCG-3') used in these experiments and for sequencing (data not shown) were complimentary to sequences near the 5 ' end of the predicted mRNA sequence and radioactively end-labeled with $\left[\gamma^{32} \mathrm{P}\right]$ ATP and T4 polynucleotide kinase [50]. In each primer extension assay, the same primer was also used for a concurrent dideoxy sequencing reaction [56]. For primer extension, total RNA was isolated from strain NA1000 (O.D. at $600 \mathrm{~nm}$ of 1.0) grown in M2 glucose minimal medium. Pelleted cells were resuspended in 20 mM sodium acetate, $1 \mathrm{mM}$ EDTA ( $\mathrm{pH}$ 5.4). 10\% SDS was then added to a final concentration of $4 \%$, followed by addition of an equal volume of phenol equilibrated with $20 \mathrm{mM}$ sodium acetate, $1 \mathrm{mM}$ EDTA ( $\mathrm{pH} 5.4$ ). Extraction, consisting of vortexing and incubation at $65^{\circ} \mathrm{C}$ for 10 minutes, was performed twice. The RNA was then ethanol precipitated from the aqueous phase, rinsed twice with $70 \%$ ethanol, and dried. Approximately $50 \mu \mathrm{g}$ of the isolated RNA was used for primer extension.

In vitro transcription was performed as described [50]. The template was a cesium chloride gradient-purified preparation of pBluescript KS+ containing either the $900 \mathrm{bp}$ gyrBP3 promoter fragment (see Fig. 3) or an approximately 500 bp PstI/StuI fragment (gyrB500) containing the promoter and regions further upstream (Table 1). It was transcribed by either E. coli RNA Polymerase $\sigma^{70}$ holoenzyme (Epicenter Technologies, Madison, WI). The resultant mRNA was then reverse transcribed from the PE2 primer, as detailed above.

\section{Gel mobility shift assays}

The crude extracts used in these experiments were prepared from mid-log phase, 0.5-liter cultures of wild-type Caulobacter crescentus (NA1000). The cells were collected by centrifugation and then rinsed three to four times with HEMGK (20 mM HEPES, pH 7.6; 0.1 mM EDTA, pH 8.0; $12.5 \mathrm{mM} \mathrm{MgCl}$; $5 \%$ glycerol; $100 \mathrm{mM} \mathrm{KCl}$ ) before being re-suspended in $4 \mathrm{ml}$ of the same. PMSF in 95\% ethanol was then added to $0.1 \%$ and the cells were lysed by sonication. The lysate was cleared by centrifugation at 34,500 $\times \mathrm{g}$ in a Sorvall SS-34 rotor; the cleared lysate, containing approximately 1 to $3 \mathrm{mg} / \mathrm{ml}$ protein, was immediately frozen $\left(-80^{\circ} \mathrm{C}\right)$ in $0.4 \mathrm{ml}$ aliquots. Unless otherwise indicated, each aliquot was thawed for use only once. The DNA probe was prepared by digesting pKSgyrB500 with 
HindIII (a site derived from the polylinker) and PstI, purifying the fragment from a $1 \%$ agarose gel and then labeling with $\left[\alpha-{ }^{32} \mathrm{P}\right]$ dGTP by filling in the HindIII site with DNA polymerase I, Klenow fragment (Promega). The gel shift assays were performed essentially as described, with some minor modifications [60]. Briefly, each reaction contained $10-20 \times 10^{3} \mathrm{cpm}$ of the labeled DNA probe and was incubated in Incubation Buffer $(20 \mathrm{mM}$ Tris-Cl, $\mathrm{pH}$ $7.5 ; 80 \mathrm{mM} \mathrm{NaCl} ; 1 \mathrm{mM}$ EDTA, $\mathrm{pH} 8.0 ; 0.1 \mathrm{mg} / \mathrm{ml}$ salmon-sperm DNA, sonicated) for 10 minutes at room temperature, after which loading dye was added and the samples run on a $6 \%$ non-denaturing polyacrylamide gel at $4{ }^{\circ} \mathrm{C}$. Where antibody to RNA polymerase [59] was included in the reaction, 1 to $3 \mu$ l were added and all samples were incubated an additional 5 minutes at room temperature.

\section{Authors' contributions}

J.C.E. carried out the experimental portion of this study. J.C.E. and J.W.G. conceived of the study and wrote the manuscript.

\section{Acknowledgements}

J.C.E. was supported by USPHS predoctoral fellowship GM-07 I85. Work in our laboratory is supported by Public Health Service Grant GM484I7 from the National Institutes of Health to J.W.G.

\section{References}

I. Ausmees N, Jacobs-Wagner C: Spatial and temporal control of differentiation and cell cycle progression in Caulobacter crescentus. Annu Rev Microbiol 2003, 57:225-247.

2. Ryan KR, Judd EM, Shapiro L: The CtrA response regulator essential for Caulobacter crescentus cell-cycle progression requires a bipartite degradation signal for temporally controlled proteolysis. I Mol Biol 2002, 324:443-455.

3. England JC, Gober JW: Cell cycle control of cell morphogenesis in Caulobacter. Curr Opin Microbiol 200I, 4:674-680.

4. Gober JW, England JC: Regulation of flagellum biosynthesis and motility in Caulobacter,. In Prokaryotic Development Edited by: Brun YV, Shimkets LJ. American Society for Microbiology, Washington, D. C. 2000:319-339.

5. Quon KC, Marczynski GT, Shapiro L: Cell cycle control by an essential bacterial two-component signal transduction protein. Cell 1996, 84:83-93.

6. Quon KC, Yang B, Domian IJ, Shapiro L, Marczynski GT: Negative control of bacterial DNA replication by a cell cycle regulatory protein that binds at the chromosome origin. Proc Natl Acad Sci USA 1998, 95:120-125.

7. Domian IJ, Quon KC, Shapiro L: Cell type-specific phosphorylation and proteolysis of a transcriptional regulator controls the GI-to-S transition in a bacterial cell cycle. Cell 1997, 90:415-424.

8. Domian IJ, Reisenauer A, Shapiro L: Feedback control of a master bacterial cell-cycle regulator. Proc Natl Acad Sci USA 1999, 96:6648-6653.

9. Marczynski GT, Shapiro L: Cell-cycle control of a cloned chromosomal origin of replication from Caulobacter crescentus. Mol Biol 1992, 226:959-977.

10. Marczynski GT, Lentine K, Shapiro L: A developmentally regulated chromosomal origin of replication uses essential transcription elements. Genes Dev 1995, 9:1543-1557.

II. Kelly AJ, Sackett MJ, Din N, Quardokus E, Brun YV: Cell cycledependent transcriptional and proteolytic regulation of FtsZ in Caulobacter. Genes Dev 1998, I 2:880-893.
12. Laub MT, Chen SL, Shapiro L, McAdams HH: Genes directly controlled by CtrA, a master regulator of the Caulobacter cell cycle. Proc Natl Acad Sci USA 2002, 99:4632-4637.

13. Laub MT, McAdams HH, Feldblyum T, Fraser CM, Shapiro L: Global analysis of the genetic network controlling a bacterial cell cycle. Science 2000, 290:2।44-2|48.

14. Roberts RC, Shapiro L: Transcription of genes encoding DNA replication proteins is coincident with cell cycle control of DNA replication in Caulobacter crescentus. I Bacteriol 1997, I 79:2319-2330.

15. Winzeler E, Shapiro L: A novel promoter motif for Caulobacter cell cycle-controlled DNA replication genes. J Mol Biol 1996 , 264:4I 2-425.

16. Zweiger G, Shapiro L: Expression of Caulobacter dnaA as a function of the cell cycle. J Bacteriol 1994, I 76:40 I-408.

17. Rizzo MF, Shapiro L, Gober JW: Asymmetric expression of the Gyrase B gene from the replication-competent chromosome in the Caulobacter crescentus predivisional cell. Bacteriol 1993, I 75:6970-698|

18. Gomes S, Gober JW, Shapiro L: Expression of the Caulobacter heat shock gene dnaK is developmentally controlled during growth at normal temperatures. J Bacteriol 1990, I72:3051-3059.

19. Malakooti J, Wang SP, Ely B: A consensus promoter sequence for Caulobacter crescentus genes involved in biosynthetic and housekeeping functions. J Bacteriol 1995, 177:4372-4376.

20. Gober JW, Shapiro L: Temporal and spatial regulation of developmentally expressed genes in Caulobacter. BioEssays 1991, I 3:277-283.

21. Evinger M, Agabian N: Caulobacter crescentus nucleoid: analysis of sedimentation behavior and protein composition during the cell cycle. Proc Natl Acad Sci USA 1979, 76: I75-I78.

22. Evinger M, Agabian N: Envelope-associated nucleoid from Caulobacter crescentus stalked and swarmer cell. J Bacteriol I977, I 32:294-30 I

23. Swoboda KK, Dow CS, Vitkovic L: Nucleoids of Caulobacter crescentus CB I 5. J Gen Microbiol 1982, I 28:279-289.

24. Keiler KC, Shapiro L: Conserved promoter motif is required for cell cycle timing of dnaX transcription in Caulobacter. J Bacteriol 200 I, I 83:4860-4865.

25. Avedissian M, Lessing D, Gober JW, Shapiro L, Gomes SL: Regulation of the Caulobacter crescentus dnaKJ operon. I Bacteriol 1995, I 77:3479-3484.

26. Malakooti J, Ely B: Principal sigma subunit of the Caulobacter crescentus RNA Polymerase. I Bacteriol 1995, I 77:6854-6860.

27. Perez-Martin J, de Lorenzo V: Clues and consequences of DNA bending in transcription. Annu Rev Microbiol 1997, 5 I:593-628.

28. Aiyar SE, Gourse RL, Ross W: Upstream A-tracts increase bacterial promoter activity through interactions with the RNA polymerase $\alpha$ subunit. Proc Natl Acad Sci USA 1998 , 95: | 4652-| 4657

29. Wolf SG, Frenkiel D, Arad T, Finkel SE, Kolter R, Minsky A: DNA protection by stress-induced biocrystallization. Nature 1999 , 400:83-85.

30. Antelmann H, Engelmann S, Schmid R, Sorokin A, Lapidus A, Hecker $M$ : Expression of a stress- and starvation-induced dps/pexBhomologous gene is controlled by the alternative sigma factor $\sigma^{\mathbf{B}}$ in Bacillus subtilis. J Bacteriol 1997, I 79:725।-7256.

31. Martinez A, Kolter R: Protection of DNA during oxidative stress by the nonspecific DNA-binding protein Dps. J Bacteriol 1997, 179:5188-5194.

32. Almirón M, Link AJ, Furlong D, Kolter R: A novel DNA-binding protein with regulatory and protective roles in starved Escherichia coli. Genes Dev 1992, 6:2646-2654.

33. Hirano T: The ABCs of SMC proteins: two-armed ATPases for chromosome condensation, cohesion, and repair. Genes Dev 2002, 16:399-4|4.

34. Graumann PL: SMC proteins in bacteria: condensation motors for chromosome segregation? Biochimie 200I, 83:53-59.

35. Cobbe N, Heck MMS: Review: SMCs in the world of chromosome biology - from prokayotes to higher eukaryotes. J Struct Biol 2000, I 29: 123-143.

36. Hirano T: SMC-mediated chromosome mechanics: a conserved scheme from bacteria to vertebrates? Genes Dev 1999 , 13:11-19. 
37. Strunnikov AV, Jessberger R: Structural maintenance of chromosomes (SMC) proteins: conserved molecular properties for multiple biological functions. Eur J Biochem 1999, 263:6-13.

38. Graumann PL, Losick R, Strunnikov AV: Subcellular localization of Bacillus subtilis SMC, a protein involved in chromosome condensation and segregation. J Bacteriol 1998, 180:5749-5755.

39. Azam TA, Iwata A, Nishimura A, Ueda S, Ishihama A: Growth phase-dependent variation in protein composition of the Escherichia coli nucleoid. J Bacteriol 1999, 181:636I-6370.

40. McLeod SM, Johnson RC: Control of transcription by nucleoid proteins. Curr Opin Microbiol 200I, 4:152-159.

4I. Falconi M, Colonna B, Prosseda G, Micheli G, Gualerz CO: Thermoregulation of Shigella and Escherichia coli EIEC pathogenicity: a temperature-dependent structural transition of DNA modulates accessibility of virF promoter to transcriptional repressor H-NS. EMBO J 1998, 17:7033-7043.

42. Dorman CJ, Deighan P: Regulation of gene expression by histone-like proteins in bacteria. Curr Opin Genet Dev 2003, 13:179-184.

43. Oberto J, Drlica K, Rouvière-Yaniv J: Histones, HMG, HU, IHF: Même combat. Biochimie 1994, 76:901-908.

44. Britton RA, Lin DC-H, Grossman AD: Characterization of a prokaryotic SMC protein involved in chromosome partitioning. Genes Dev 1998, 12:1254-1259.

45. Moriya S, Tsujikawa E, Hassan AK, Asai M, Kodama KT, Ogasawara $\mathrm{N}$ : A Bacillus subtilis gene-encoding protein homologous to eukaryotic SMC motor protein is necessary for chromosome partition. Mol Microbiol 1998, 29: I78-187.

46. Jensen RB, Shapiro L: The Caulobacter crescentus smc gene is required for cell cycle progression and chromosome segregation. Proc Natl Acad Sci USA 1999, 96: I066 I-10666.

47. Altuvia S, Almiron M, Huisman G, Kolter R, Stortz G: The dps promoter is activated by OxyR during growth and by IHF and $\sigma^{\text {s }}$ in stationary phase. Mol Microbiol 1994, 13:265-272.

48. Gober JW, Shapiro L: A developmentally regulated Caulobacter flagellar promoter is activated by $3^{\prime}$ enhancer and IHF binding elements. Mol Biol Cell 1992, 3:913-926.

49. Simons R, Priefer U, Puhler A: A broad host range mobilization system for in vivo genetic engineering: transposon mutagenesis in gram negative bacteria. Bio/Technology 1983, I:784-790.

50. Sambrook J, Fritsch EF, Maniatis T: Molecular cloning: a laboratory manual 2 nd edition. Cold Spring Harbor Laboratory Press, Cold Spring Harbor, New York; 1989.

5I. Poindexter JS: Biological properties and classification of the Caulobacter group. Bacteriol Rev 1964, 28:23 I-295.

52. Johnson RC, Ely B: Isolation of Spontaneously Derived Mutants of Caulobacter crescentus. Genetics 1977, 86:25-32.

53. Ely B, Johnson RC: Generalized transduction in Caulobacter crescentus. Genetics 1977, 87:391-399.

54. Schweizer HP: Allelic exchange in Pseudomonas aeruginosa using novel ColE I-type vectors and a family of cassettes containing a portable oriT and the counter-selectable Bacillus subtilis sacB marker. Mol Microbiol 1992, 6: I | 95- I 204.

55. Alexeyev MF: The pKNOCK series of broad-host-range mobilizable suicide vectors for gene knockout and targeted DNA insertion into the chromosome of gram-negative bacteria. Biotechniques 1999, 26:824-828.

56. Kunkel TA, Robert JD, Zakour RA: Rapid and efficient site-specific mutagenesis without phenotypic selection. Methods Enzymol 1987, I 54:367-382.

57. Sanger $F$, Nicklen $S$, Coulson AR: DNA sequencing with chainterminating inhibitors. Proc Natl Acad Sci USA 1977 , 74:5463-5467.

58. Mangan EK, Bartamian M, Gober JW: A mutation that uncouples flagellum assembly from transcription alters the temporal pattern of flagellar gene expression in Caulobacter crescentus. J Bacteriol 1995, 177:3176-3184.

59. Miller JH: Experiments in molecular genetics. Cold Spring Harbor Laboratory, Cold Spring Harbor, NY; 1972.

60. Amemiya K, Bellofatto V, Shapiro L, Feingold J: Transcription initiation in vitro and in vivo at a highly conserved promoter within a 16 S ribosomal RNA gene. I Mol Biol 1986, 187:1-14.

61. Ausubel FM, Brent R, Kingston RE, Moore DD, Seidman JG, Smith JA, Struhl K: Current Protocols in Molecular Biology. John Wiley and Sons, Inc., New York, NY; 1996.
62. Carter P, Beduouelle H, Winter G: Improved oligonucleotide site-directed mutagenesis using MI 3 vectors. Nucleic Acids Res 1985, 13:443|-4443.
Publish with Bio Med Central and every scientist can read your work free of charge

"BioMed Central will be the most significant development for disseminating the results of biomedical research in our lifetime. "

Sir Paul Nurse, Cancer Research UK

Your research papers will be:

- available free of charge to the entire biomedical community

- peer reviewed and published immediately upon acceptance

- cited in PubMed and archived on PubMed Central

- yours - you keep the copyright 